\title{
High current nonlinear transmission line based electron beam driver
}

\author{
B. W. Hoff, ${ }^{1}$ D. M. French, ${ }^{1}$ D. S. Simon, ${ }^{1}$ P. D. Lepell, ${ }^{2}$ T. Montoya, ${ }^{3}$ and S. L. Heidger ${ }^{1}$ \\ ${ }^{1}$ Air Force Research Laboratory, Directed Energy Directorate, Kirtland AFB, New Mexico 87117, USA \\ ${ }^{2}$ Leidos, 2109 Air Park Road SE, Albuquerque, New Mexico 87106, USA \\ ${ }^{3}$ Voss Scientific, 418 Washington Street SE, Albuquerque, New Mexico 87108, USA
}

(Received 17 July 2017; published 12 October 2017)

\begin{abstract}
A gigawatt-class nonlinear transmission line based electron beam driver is experimentally demonstrated. Four experimental series, each with a different Marx bank charge voltage (15, 20, 25, and $30 \mathrm{kV})$, were completed. Within each experimental series, shots at peak frequencies ranging from $950 \mathrm{MHz}$ to $1.45 \mathrm{GHz}$ were performed. Peak amplitude modulations of the NLTL output voltage signal were found to range between $18 \%$ and $35 \%$ for the lowest frequency shots and between $5 \%$ and $20 \%$ for the highest frequency shots (higher modulation at higher Marx charge voltage). Peak amplitude modulations of the electron beam current were found to range between $10 \%$ and $20 \%$ for the lowest frequency shots and between $2 \%$ and $7 \%$ for the highest frequency shots (higher modulation at higher Marx charge voltage).
\end{abstract}

DOI: 10.1103/PhysRevAccelBeams.20.100401

\section{INTRODUCTION}

High current electron beams are applicable to a variety of technology areas, including the generation of electromagnetic radiation, such as microwaves and $\mathrm{x}$ rays, particle accelerators, and material processing and joining techniques, such as welding. In applications in which a modulated electron beam is desirable or required, there exist a number of well-established methods, such as the utilization of gated cathodes, ferroelectric emission cathodes, and photocathodes [1-7] that enable modulation of the electron beam at the point of emission. Another more recently demonstrated technique to allow for the emission of a modulated electron beam used a field emission cathode driven by the output of a nonlinear transmission line (NLTL) [8,9]. In the experiments described in Ref. [9], French et al. generated modulated electron beams ranging from 1 to $3 \mathrm{~mA}$ of average current with peak voltages around $1 \mathrm{kV}$, demonstrating the concept of a NLTL-based beam modulator at low power.

Because a NLTL-based beam modulation system might be incorporated into the pulsed power system driving an electron beam diode, use of a NLTL beam modulator in some applications may allow for increased system compactness. It is noted, however, that due to the relatively short rf pulse durations from typical high power NLTLs [10-14], likely applications for a NLTL-based beam modulation system would be those requiring short (1's to 10's of ns) modulated bursts of electrons as opposed to

Published by the American Physical Society under the terms of the Creative Commons Attribution 4.0 International license. Further distribution of this work must maintain attribution to the author(s) and the published article's title, journal citation, and DOI. continuous or quasi-continuous emission of a modulated electron beam.

Results from simulation work published previously by Hoff et al. [15], using the particle-in-cell code icepic [16], predict the ability to use a NLTL to drive kA-class beams at average voltages of 100's of $\mathrm{kV}$ with current modulation levels exceeding $30 \%$ of the average beam current. As described in Ref. [15], the NLTL itself was not modeled as part of the beam driver; instead, measured voltage waveforms from a previously developed high power NLTL experiment [17] were used as the input signals. These signals were injected via a short section of a transmission line connected to a cathode which was able to launch the electron beam down a magnetized drift tube. A depiction of a radial slice of the previously simulated [15] geometry is provided in Fig. 1(a). Also shown, in Fig. 1(b), is a pseudocolor plot of the axial magnetic field magnitude produced by the magnet coils included in the model. The tapered field profile is required for operation of the Friedman type cathode configuration [18] used in the simulations.

\section{EXPERIMENTAL CONFIGURATION}

In the present paper, the authors report on experimental results from a high power NLTL-modulated electron beam diode. The electron beam diode and drift tube geometry used in these experiments were based on those modeled by Hoff et al. in Ref. [15], as previously discussed. A schematic of the experimental configuration, including specific diagnostics and data channels, is depicted in Fig. 2. The 15 stage Marx pulser and NLTL used in the experiment were the same as those reported on in Ref. [17] and were configured in the same manner, with the exception that for the present experiments, the output of the NLTL was connected to the beam driver assembly instead of to the $50 \Omega$ transmission line used by French et al. Four 


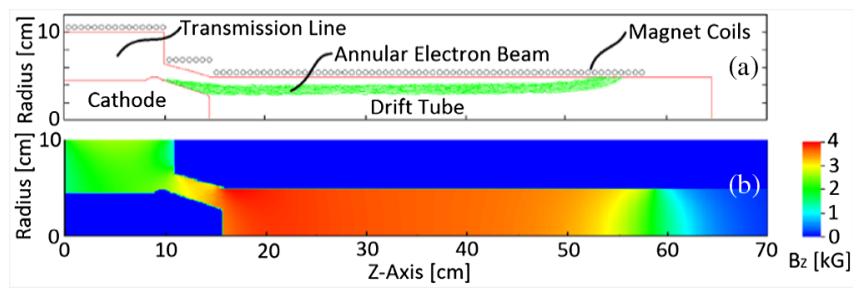

FIG. 1. Geometry (a) and magnetic field magnitude profile (b) for 2D simulations of the NLTL-based electron beam driver.

series of experiments, using Marx bank charge voltages of $15,20,25$, and $30 \mathrm{kV}$, respectively, were performed. Voltage waveforms from the output of the NLTL were measured using a calibrated D-dot probe located at L-C Stage 58, just before the output coupling of the NLTL [17].

A machine drawing of the electron beam gun is shown in Fig. 3. The gun consisted of a section of $50 \Omega$ tapered transmission line that begins at the output coupling of the NLTL and terminates at the cathode. The same Friedman cathode [14] geometry modeled in Ref. [15] was used in the experiment. The emission region of the graphite cathode, indicated in Fig. 3, was coated with carbon fibers [19]. The cathode emission region had an axial length of four centimeters, starting with an outer radius equal to that of the coaxial transmission line inner conductor radius of $3.50 \mathrm{~cm}$, tapered to a radius of $2.64 \mathrm{~cm}$. The cathode surface is separated from the anode by a $2 \mathrm{~cm}$ gap. Immediately upstream of the cathode is a toroidal field shaping bump intended to suppress backstreaming of the electrons emitted from the cathode.

The vacuum drift tube started at the downstream end of the cathode and extended for $90 \mathrm{~cm}$, after which the drift tube transitioned to a tapered beam dump. The drift tube had an inner radius of $5.5 \mathrm{~cm}$. The downstream end of the drift tube was connected to a tapered beam dump which was, in turn, connected to the inlet of a small vacuum cryo-pump. Vacuum levels of $\sim 1 \times 10^{-7}$ Torr were maintained throughout the operation of the experiment. The drift tube was designed with four diagnostic ports (1A, 2A, 3A, and 4A), spaced

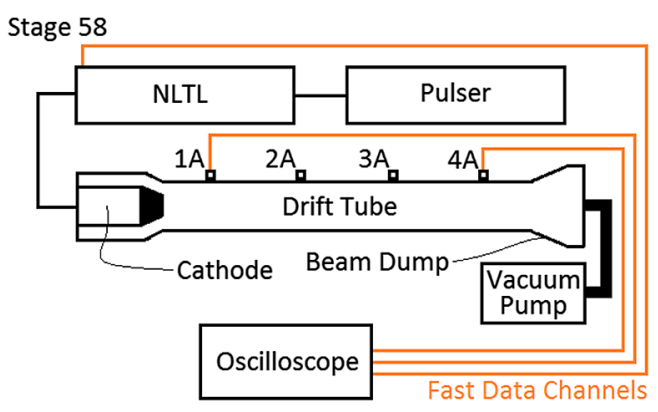

FIG. 2. Schematic of the NLTL beam driver experiment, including specified diagnostics, such as the Stage 58 D-dot, $1 \mathrm{~A}-4 \mathrm{~A}$ B-dots, and fast data channels for sampling of $\mathrm{rf}$ waveform data.

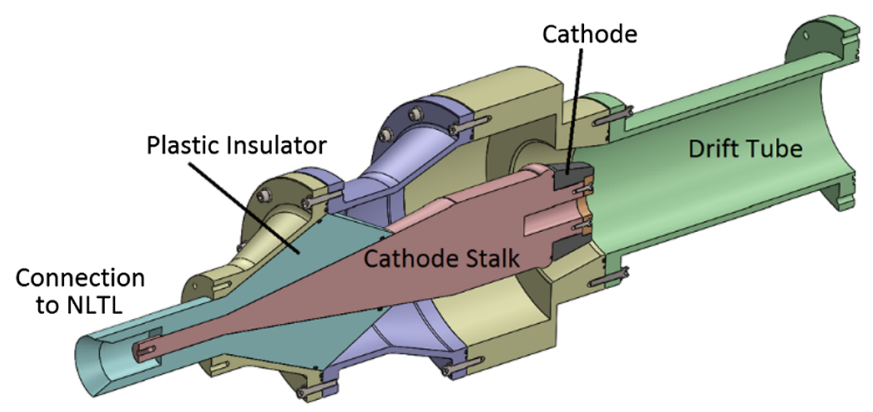

FIG. 3. Machine drawing of the electron beam diode region. A shortened drift tube is depicted to allow for a better aspect ratio to be used in the figure.

equidistantly along the wall of the drift tube. Ports $1 \mathrm{~A}$ (an axial distance of $20 \mathrm{~cm}$ from the end of the cathode) and $4 \mathrm{~A}$ (an axial distance of $71 \mathrm{~cm}$ from the end of the cathode) were populated with calibrated B-dot probes for measurement of the beam current waveforms.

A labeled photo of the electron beam diode, solenoidwrapped vacuum drift tube, and beam dump is provided in Fig. 4. The solenoid coils, wrapped directly on the outer wall of the drift tube, include four booster coils which were located above the gaps in the coil necessitated by the $1 \mathrm{~A}-4 \mathrm{~A}$ diagnostic ports. Figure 5(a) shows a slice of the simulated coil configuration overlaid with a plot of the magnetic field magnitude (red represents higher field regions; blue represents lower field regions). A plot of measured axial magnetic field values along the length of the solenoid at a radius of $4 \mathrm{~cm}$ (the predicted beam radius) is provided in Figure 5(b). The measured values are overlaid with the simulated axial magnetic field values. The as-built coils generate a magnetic field with larger distortions near the diagnostic port locations than was predicted in simulation, but this was not expected to preclude transport of the beam down the drift tube. The magnetic field coils are energized with a long pulse voltage source. Due to limitations of the drift tube solenoid pulser at the time of the experiment, the pulser was capable of driving a maximum of $1.15 \mathrm{kA}$ through the drift tube coils during operation of the experiment instead of the design current of $1.5 \mathrm{kA}$ used in the ICEPIC simulations [15].

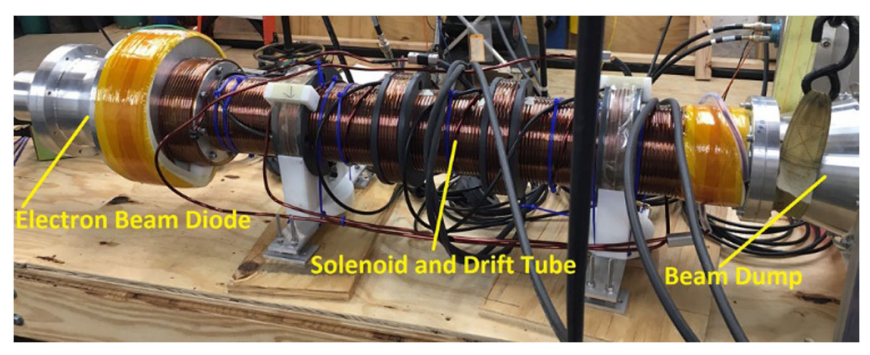

FIG. 4. Photo of the electron beam diode, solenoid-wrapped drift tube, and beam dump. 


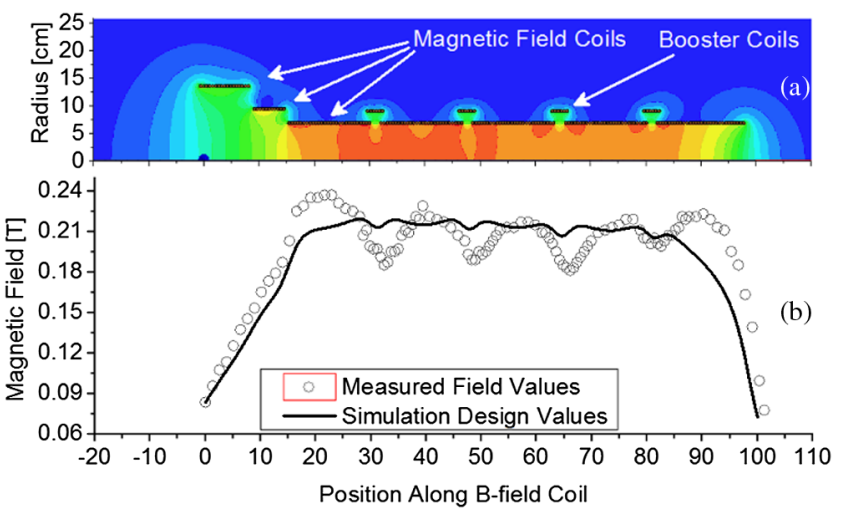

FIG. 5. Slice of the simulated coil configuration, overlaid with a pseudocolor plot of the magnetic field magnitude (a), and the magnetic field measured along the length of the drift tube at a radius of $4 \mathrm{~cm}$, overlaid with the corresponding simulation values (b). Magnetic field simulations were performed using Ansys Maxwell.

\section{EXPERIMENTAL RESULTS AND ANALYSIS}

A detailed analysis is shown for a single shot from the $25 \mathrm{kV}$ Marx charge experiment series which is used to describe the analysis technique. This single shot analysis is followed by a multishot analysis of all shots in each of the four experimental series $(15,20,25$, and $30 \mathrm{kV})$ performed.

\section{A. Single shot results and analysis}

NLTL Stage 58 D-dot voltage data as well as current data measured at B-dots $1 \mathrm{~A}$ and $4 \mathrm{~A}$ for an example shot (Shot 20) from the $25 \mathrm{kV}$ Marx charge experimental series are presented in Fig. 6. The time axes of each of the subtraces (NLTL Stage 58 D-dot, B-dot 1A, and B-dot 4A) are shifted such that the rising edge of each is aligned for ease of comparison. It is also noted that the negative time values are simply an artifact of the way triggering was

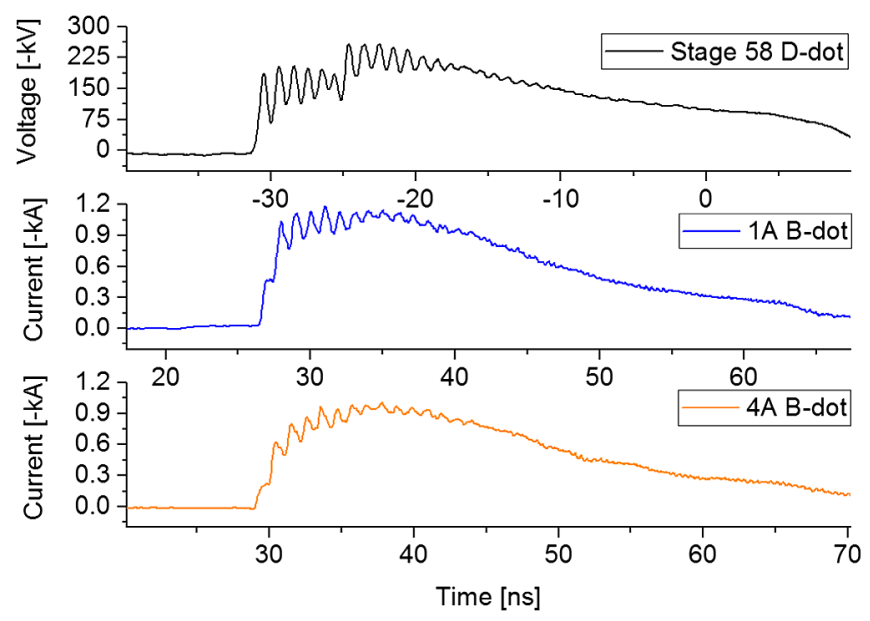

FIG. 6. Voltage trace measured at the NLTL Stage 58 D-dot and beam current traces measured at the $1 \mathrm{~A}$ and $4 \mathrm{~A} \mathrm{~B}$-dot locations for Shot 20 of the $25 \mathrm{kV}$ series. performed in the experiment and that only the relative time values between the subplots is meaningful.

Inspection of the NLTL Stage 58 D-dot voltage trace in Fig. 6 shows a step in the quasi-dc portion of the signal, indicative of an impedance mismatch between the transmission line and the cathode. Applying a low pass filter with a cutoff frequency of $500 \mathrm{MHz}$ to the signal data plotted in Fig. 6 removes the majority of the rf portion of the signal, leaving the remaining quasi-DC portion. The low pass filtered data are plotted in Fig. 7. The aforementioned voltage step in the NLTL Stage 58 D-dot signal is readily observable in the filtered NLTL Stage 58 D-dot trace.

The effective impedance of the electron beam diode, for this shot, can be estimated in the following manner. As described in Ref. [17], the saturated output of the NLTL is approximately $50 \Omega$. The short transmission line connection, taper, and vacuum transition sections connecting the output of the NLTL to the electron beam diode section (shown in Fig. 3) were also designed to be approximately $50 \Omega$. Thus, for the purposes of this calculation, the transmission line impedance, $Z_{0}$, is assumed to be exactly $50 \Omega$. The voltage of the pulse leaving the NLTL is approximately $150 \mathrm{kV}$, and the voltage of the reflected pulse is $75 \mathrm{kV}(225-150 \mathrm{kV})$. For transmission line systems, the reflection coefficient, $\Gamma$, can be defined as

$$
\Gamma=\frac{V^{-}}{V^{+}},
$$

which in the present calculation is equal to 0.5 . From this reflection coefficient the impedance of the transmission line load, $Z_{L}$ (the electron beam diode), can be determined as

$$
Z_{L}=Z_{0}\left(\frac{1+\Gamma}{1-\Gamma}\right)
$$

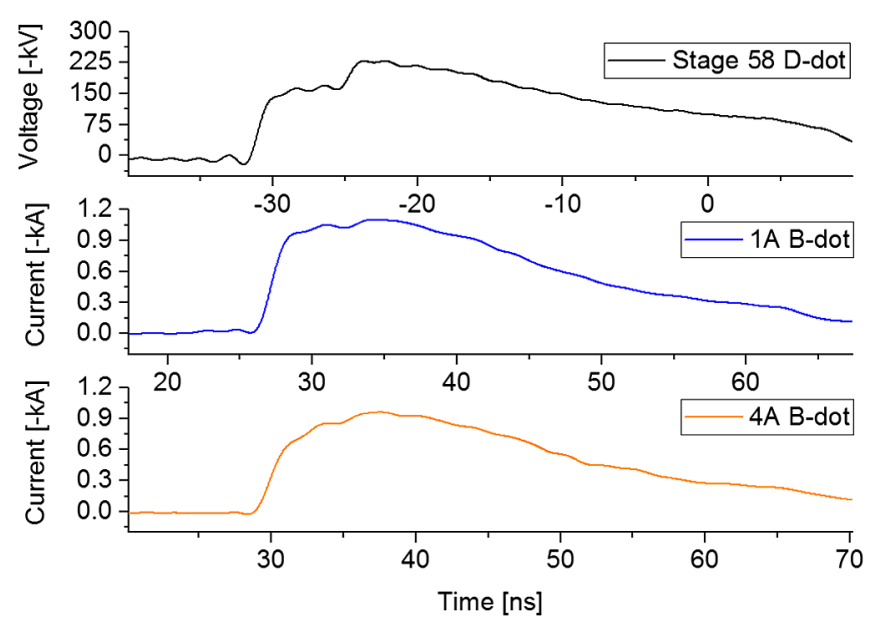

FIG. 7. Low-pass filtered (500 MHz cutoff) voltage and current traces from Shot 20 of the $25 \mathrm{kV}$ series (unfiltered waveforms shown in Fig. 6). 
and is approximately equal to $150 \Omega$. Based on this impedance, the expected cathode current is $\sim 1.5 \mathrm{kA}$ $(225 \mathrm{kV} / 150 \Omega)$. The leading portion of the measured filtered beam current pulse at B-dot $1 \mathrm{~A}$ is approximately $950 \mathrm{~A}$, which indicates that around $37 \%$ of the current is lost in the diode region and does not travel downstream. It is noted, however, that despite the differences in appearance between the 1A and 4A B-dot traces in Figs. 6 and 7, direct integration of the full current traces yield better than $95 \%$ agreement in total charge, indicating that the beam is being efficiently transported down the drift tube.

Application of high pass filtering of the waveforms in Fig. 6, with a cutoff frequency of $500 \mathrm{MHz}$, allows inspection of the rf components of each of the signals and results in the traces plotted in Fig. 8. As is noted from comparison of the NLTL Stage 58 D-dot voltage, strong rf oscillations appear to last for approximately twice as long as in the current traces; however, recalling the previous discussion regarding the measured reflections back into the transmission line from the higher impedance electron beam diode, it is reasonable to assume that the majority of the rf signal amplitude in the NLTL Stage 58 D-dot trace after approximately $t=-25 \mathrm{~ns}$ is due to measurement of the reflected pulse. A comparison of the $1 \mathrm{~A}$ and $4 \mathrm{~A}$ B-dot current traces shows a reduction of the amplitude of the $\mathrm{rf}$ signal, indicating a degree of demodulation occurring as the beam travels down the drift tube.

Data from fast Fourier transform (FFT) analysis of the Fig. 8 waveforms are presented in Fig. 9. The peak frequency of the rf signal shifts lower in frequency downstream of the electron beam diode and then is further downshifted as the beam traverses the length of the drift tube. The initial reduction in frequency occurring between the NLTL Stage 58 D-dot location and B-dot $1 \mathrm{~A}$ is potentially a result of filtering taking place because of

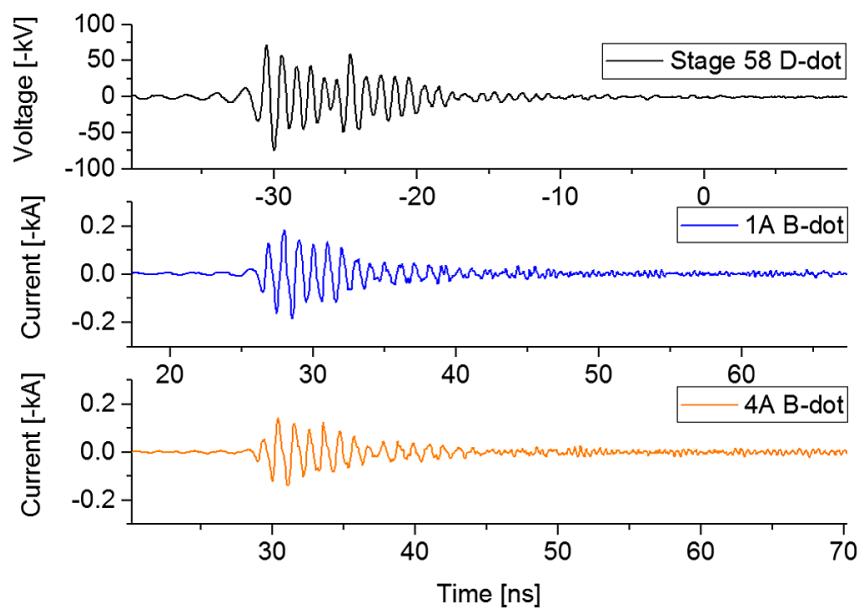

FIG. 8. High-pass filtered (500 MHz cutoff) voltage and current traces from Shot 20 of the $25 \mathrm{kV}$ series (unfiltered waveforms shown in Fig. 6). the inductive and capacitive properties of the diode. Linear kinetic bunching theory [20] predicts that the amplitude of the space-charge waves will vary sinusoidally as the beam propagates down the drift tube, with a wavelength that is inversely proportional to the frequency. In a beam with a range of frequencies such as is present in Fig. 9, there is a section of tube in which the components of the spacecharge wave with frequencies below the initial peak frequency have greater magnitude than those at the initial peak frequency. This region also corresponds to lower overall current bunching. This effect is a likely contributor to the observed reduction in peak frequency occurring between B-dot $1 \mathrm{~A}$ and B-dot 4A.

A comparison of the three traces in Fig. 9 also shows a relative increase in the amplitude of higher frequency content compared to the amplitude of the peak signal frequency. Although the drift tube lacks a slow wave structure, generation of harmonic content, such as the peak around $1.9 \mathrm{GHz}$ in the $1 \mathrm{~A}$ and $4 \mathrm{~A}$ traces and around $2.9 \mathrm{GHz}$ in the $4 \mathrm{~A}$ trace is likely a result of the charge overtaking effect described in $[21,22]$.

\section{B. Multiple shot results and analysis}

Plots of peak frequency values from FFTs of the Stage 58 D-dot, 1A B-dot, and 4A B-dot data from the 15, 20, 25, and $30 \mathrm{kV}$ Marx charge experimental series are plotted as a function of applied NLTL $\mathrm{H}$ field and presented in Figs. 10(a)-10(d). Because multiple shots were taken at each solenoid current setting and would, thus, have the same applied $\mathrm{H}$ field value, the average peak frequency for each set of shots at a given applied $\mathrm{H}$ are plotted. The majority of shot sets within each of the four Marx voltage series followed the trend that the peak frequency of the beam modulations observed at the 1A B-dot location was lower than the peak frequency of the voltage oscillations

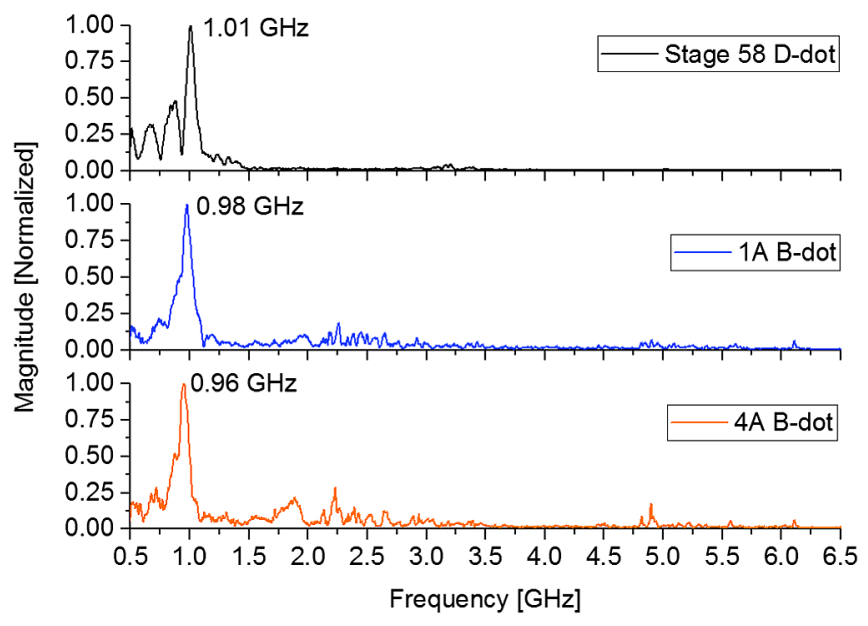

FIG. 9. FFTs of the voltage signal from the Stage 58 D-dot and the current signals from the $1 \mathrm{~A}$ and $4 \mathrm{~A}$ B-dots. 

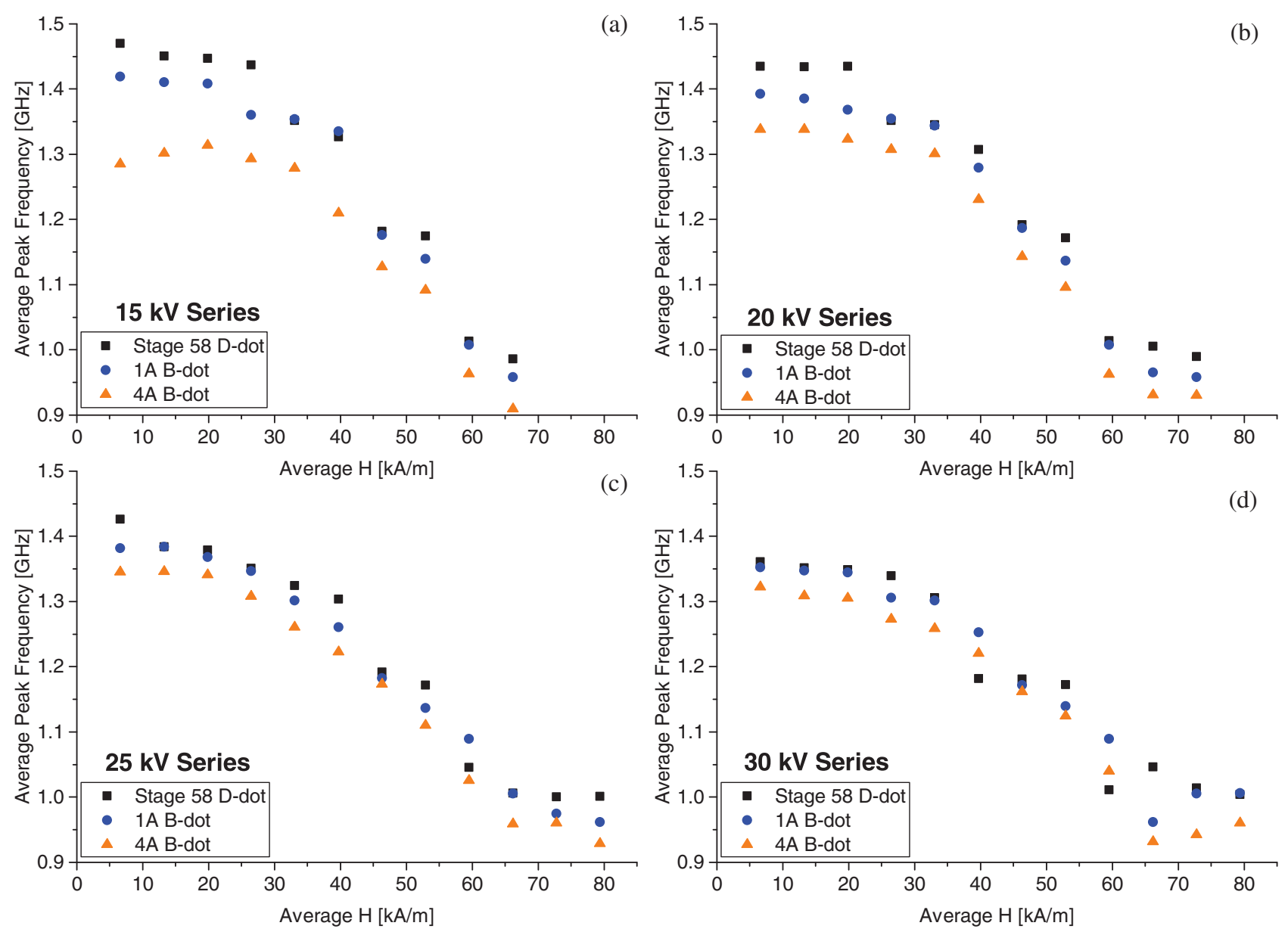

FIG. 10. Peak frequency of the Stage $58 \mathrm{D}$-dot, 1A B-dot, and 4A B-dot signals as a function of applied $\mathrm{H}$ field for the $15 \mathrm{kV}$ (a), $20 \mathrm{kV}$ (b), $25 \mathrm{kV}$ (c), and $30 \mathrm{kV}$ (d) Marx charge experimental series. In plot (a), the magnetic field biasing the NLTL is decreased as shot number is increased. Because multiple shots were taken at each solenoid current setting and would, thus, have the same applied $\mathrm{H}$ field value, the average peak frequency for each set of shots at a given applied $\mathrm{H}$ are plotted.

observed at the NLTL Stage 58 D-dot. In all cases, the peak frequency of the beam modulations observed at the 4A B-dot were lower than those observed at the 1A B-dot.

Plots of signal modulation as a function of NLTL Stage $58 \mathrm{D}$-dot peak frequency for each of the shots in the 15, 20, 25 , and $30 \mathrm{kV}$ series is provided in Fig. 11. Trend lines, based on a third order polynomial fit, are also included. Peak modulation, as used in Fig. 11, is calculated by first dividing the time series values of the high pass filtered signals (such as those depicted in Fig. 8) by the time series values of the low pass filtered signals (such as those depicted in Fig. 7). Next, a time window for each trace is set, corresponding to the period of time in which the low pass filtered signal exceeds $70 \%$ of its maximum value. Finally, the maximum rf signal amplitude ratio within the time window is selected and multiplied by 100 percentage points to yield percentage of signal modulation.

In each of the four series, the greatest NLTL voltage modulations ( $>30 \%$ in some series) were observed at the lowest frequencies. This trait of the NLTL used in these experiments has been previously documented by French et al. in Ref. [17]. Both beam current measurements (1A B-dot and 4A B-dot) also follow this trend, but, in all cases, have lower peak modulation values than the NLTL voltage signal. At lower frequencies, the difference in peak modulation between the 1A B-dot and 4A B-dot signals was found to differ by a larger margin than at higher frequencies. Because demodulation (debunching) of a modulated electron beam is enhanced both by differences in current density as well as the velocity spread of the beam [23,24], it would be expected that the demodulating effect would be strongest at the lowest frequencies which the NLTL's output waveform generates the largest voltage and current oscillations in the electron beam. The sinusoidal modulation of the space charge wave as a function of distance down the tube also plays a part in the observed reductions in peak modulation. The 4A B-dot is in a location that sees lowered frequency (Fig. 10) which is observed as the modulation of the space charge wave is in a region of decreasing amplitude. 

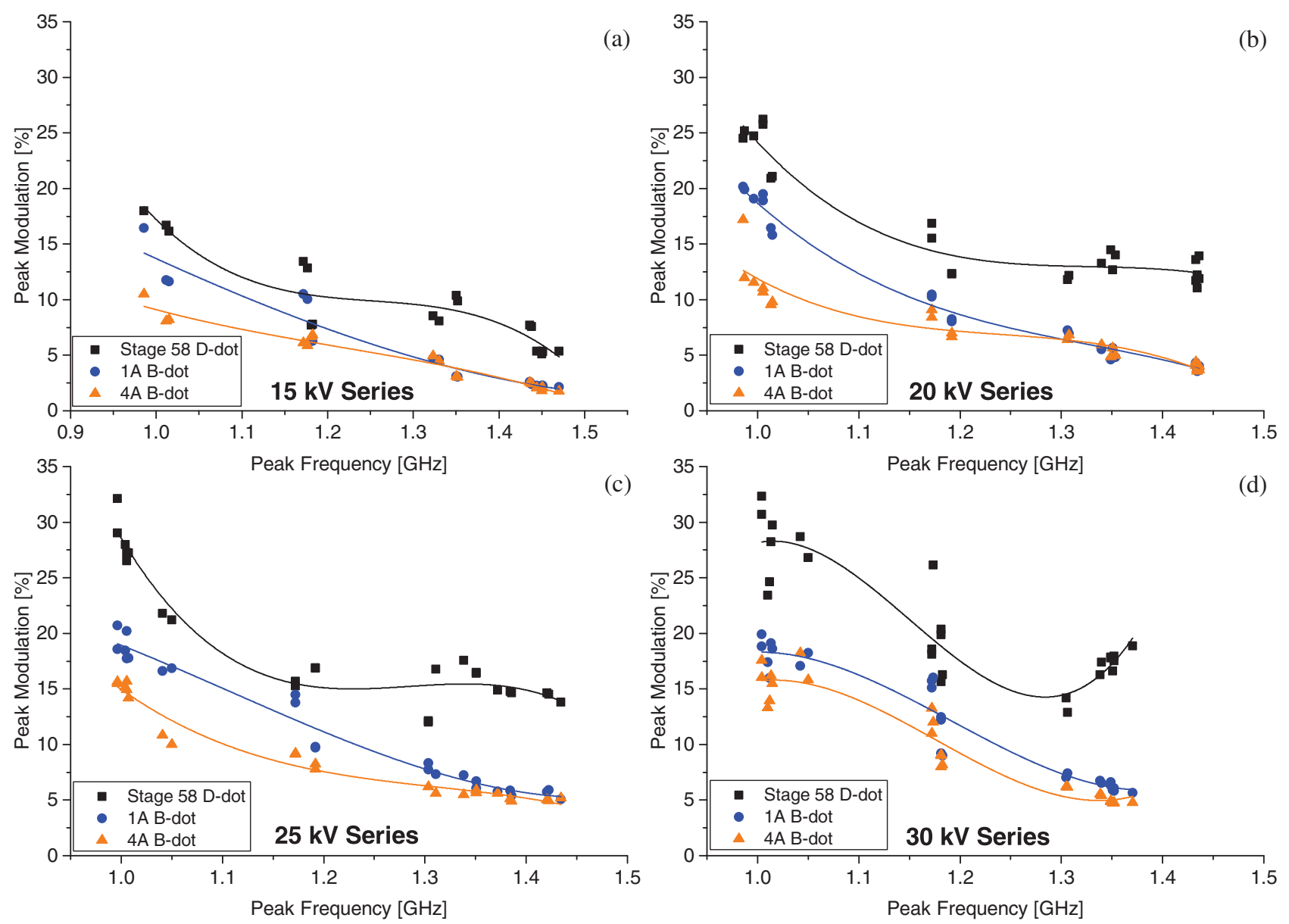

FIG. 11. Peak modulation as a function of peak frequency of the Stage 58 D-dot, 1AB-dot, and 4A B-dot signals as a function of shot number for the $15 \mathrm{kV}$ (a), $20 \mathrm{kV}$ (b), $25 \mathrm{kV}$ (c), and $30 \mathrm{kV}$ (d) Marx charge experimental series. Peak frequency values are from the Stage 58 D-dot FFT of each shot.

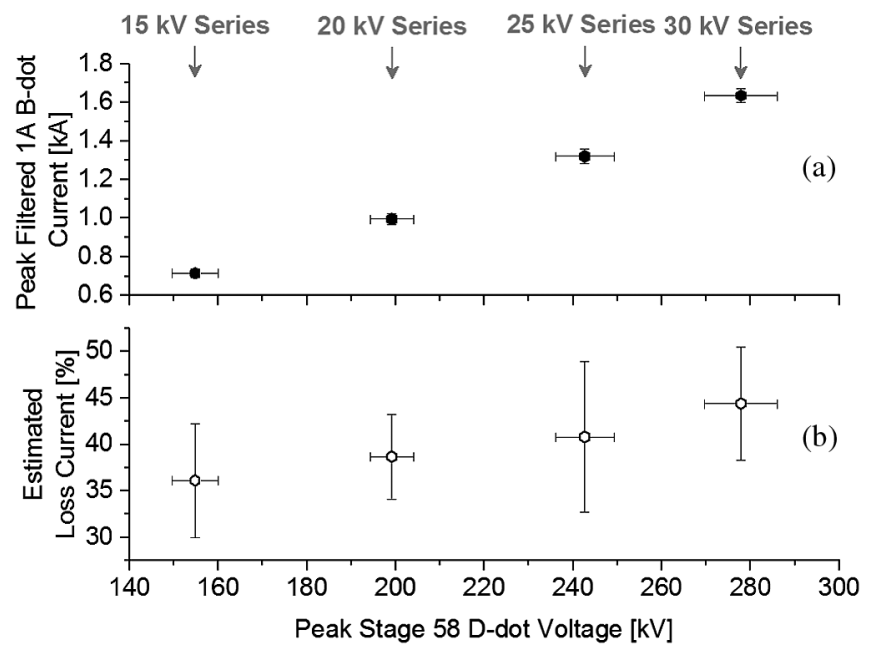

FIG. 12. Peak filtered 1A B-dot current (a) and estimated loss current (b) for the $15-30 \mathrm{kV}$ experimental series as a function of peak Stage 58 D-dot voltage.
Following the current loss estimation for the single shot case, the estimation was performed for the four experiment series. Figure 12(a) shows a plot of average peak low-pass filtered current as a function of peak filtered voltage measured at the Stage 58 D-dot. Figure 12(b) depicts a plot of estimated loss current averages for each of the four series as a function of peak filtered voltage measured at the Stage 58 D-dot. As is observed from the data, a substantial amount of current is being lost in the diode region. For axial magnetic field values in the anode-cathode gap region of the electron beam diode, shown in Fig. 5, a calculation of the Hull condition [25] indicates the diode should be magnetically insulated for voltages well beyond those achieved in the present experiment. A probable mechanism for the loss in downstream current is electron backstreaming due to the aforementioned reductions in capability of the drift tube magnet system at the time of the experiment.

\section{SUMMARY AND CONCLUSIONS}

A high current NLTL-based electron beam driver was built and tested. Four experimental series, each with a 
different Marx bank charge voltage $(15,20,25$, and $30 \mathrm{kV})$, were completed. Within each experimental series, shots at peak frequencies ranging from $950 \mathrm{MHz}$ to $1.45 \mathrm{GHz}$ were performed. Peak amplitude modulations of the NLTL voltage signal, as measured at the Stage 58 D-dot, were found to range between $18 \%$ and $35 \%$ for the lowest frequency shots and between 5\% and 20\% for the highest frequency shots (higher modulation at higher Marx charge voltage).

Peak amplitude modulations of the electron beam current, as measured at the $1 \mathrm{~A} \mathrm{~B}$-dot, were found to range between $10 \%$ and $20 \%$ for the lowest frequency shots and between $2 \%$ and $7 \%$ for the highest frequency shots (higher modulation at higher Marx charge voltage). In all cases, the current modulation measured at the 1A B-dot was lower than that for the corresponding voltage trace, measured at the NLTL Stage 58 D-dot. Additionally, some degree of demodulation of the beam was found to occur as it traveled down the drift tube from the $1 \mathrm{~A}$ B-dot location to the $4 \mathrm{~A}$ $\mathrm{B}$-dot location. At the lowest frequencies, the current modulation at the 4A B-dot was found to be as much as 8 percentage points lower than the current modulation observed at the location of the 1A B-dot. This effect was observed to become less pronounced at higher frequencies. In all series, the current modulations observed at both B-dot locations tended to coalesce to the same value. Because debunching of a modulated electron beam is enhanced both by differences in current density as well as the velocity spread of the beam, the debunching effect is expected to be strongest at the lowest frequencies (for which the NLTL's output waveform generates the largest voltage and current oscillations in the electron beam).

Comparisons between expected and measured downstream current values for each of the experimental series yielded indications of significant current loss in the diode region. Electron backstreaming due to reductions in capability of the drift tube magnet system at the time of the experiment was found to be the most likely mechanism for the discrepancies in expected versus measured downstream current. Current losses occurring during transport of the beam down the drift tube were found to be negligible.

\section{ACKNOWLEDGMENTS}

This work was supported by the Air Force Office of Scientific Research under FA9550-16RDCOR310 and FA9550-16RDCOR281. The authors note that at the time of D. M. F.'s contributions to this work, he was affiliated with the Air Force Research Laboratory.

[1] J. R. Harris and P. G. O'Shea, Gridded electron guns and modulation of intense beams, IEEE Trans. Electron Devices 53, 2824, 2006.
[2] J. W. Lewellen and J. Noonan, Field-emission cathode gating for rf electron guns, Phys. Rev. ST Accel. Beams 8, 033502 (2005).

[3] K. Chirko, V. T. Gurovich, Y. E. Krasik, O. Peleg, J. Felsteiner, and V. Bernshtam, High-frequency electron beam modulation by a ferroelectric cathode with anomalous plasma resistance, Phys. Plasmas 11, 3865 (2004).

[4] R. Ganter, K. Li, M. Dehler, J. Gobrecht, L. Rivkin, and A. Wrulich, Pulsed field emitted current from different commercial samples in the purpose of a free electron laser application, J. Vac. Sci. Technol. B 23, 680 (2005).

[5] J. R. Harris, J. G. Neumann, and P. G. O'Shea, Governing factors for production of photoemission-modulated electron beams, J. Appl. Phys. 99, 093306 (2006).

[6] S. C. Leemann, A. Streun, and A. F. Wrulich, Beam characterization for the field-emitter-array cathode-based low-emittance gun, Phys. Rev. ST Accel. Beams 10, 071302 (2007).

[7] V. T. Astrelin and S. V. Lebedev, in, 10th International Conference on High-Power Particle Beams, San Diego, CA, 1994, https://www.cambridge.org/core/journals/ laser-and-particle-beams/article/the-method-of-an-intenseelectron-beam-modulation/A368383AB29F01CB1E3A0B BA83A8D1C6.

[8] B. W. Hoff, D. M. French, D. A. Shiffler, S. L. Heidger, and W. W. Tang, Nonlinear transmission line modulated electron beam emission, United State Patent 8,766,541 (2014).

[9] D. M. French, B. W. Hoff, W. Tang, S. Heidger, J. AllenFlowers, and D. Shiffler, Nonlinear transmission line based electron beam driver, Rev. Sci. Instrum. 83, 123302 (2012).

[10] N. Seddon, C. R. Spikings, and J. E. Dolan, in Proceedings of the 16th IEEE International Pulsed Power Conference, Albuquerque, NM, 2007, http://ieeexplore.ieee.org/ abstract/document/4651931/.

[11] J. Gaudet, E. Schamiloglu, J. O. Rossi, C. J. Buchenauer, and C. Frost, in Proceedings of the 2008 International Power Modulators and High Voltage Conference, Las Vegas, 2008, http://ieeexplore.ieee.org/abstract/document/4743597/.

[12] H. Ikezi, S. S. Wojtowicz, R. E. Waltz, and D. R. Baker, Temporal contraction of solitons in a nonuniform transmission line, J. Appl. Phys. 64, 6836 (1988).

[13] J.-W. B. Bragg, J. C. Dickens, and A. A. Neuber, Material selection considerations for coaxial, ferromagnetic-based nonlinear transmission lines, J. Appl. Phys. 113, 064904 (2013).

[14] V. V. Rostov, N. M. Bykov, D. N. Bykov, A. I. Klimov, O. B. Kovalchuk, and I. V. Romanchenko, Generation of subgigawatt RF pulses in nonlinear transmission lines, IEEE Trans. Plasma Sci. 38, 2681 (2010).

[15] B. W. Hoff and D. M. French, Simulations of a disk-on-rod TWT driven by an NLTL-modulated electron beam, IEEE Trans. Plasma Sci. 44, 1265 (2016).

[16] R. J. Peterkin and J. W. Luginsland, A virtual prototyping environment for directed-energy concepts, Comput. Sci. Eng. 4, 42 (2002).

[17] D. M. French and B. W. Hoff, Spatially dispersive ferrite nonlinear transmission line with axial bias, IEEE Trans. Plasma Sci. 42, 3387 (2014).

[18] M. Friedman and M. Ury, Production and focusing of a high power relativistic annular electron beam, Rev. Sci. Instrum. 41, 1334 (1970). 
[19] D. Shiffler, M. LaCour, K. Golby, M. Sena, M. Mitchell, M. Haworth, K. Hendricks, and T. Spencer, Comparison of velvet- and cesium iodide-coated carbon fiber cathodes, IEEE Trans. Plasma Sci. 29, 445 (2001).

[20] M. Friedman, J. Krall, Y. Y. Lau, and V. Serlin, Externally modulated intense relativistic electron beams, J. Appl. Phys. 64, 3353 (1988).

[21] J. W. Gewartowski and H. A. Watson, Principles of Electron Tubes (D. Van Nostrand Company, Inc., Princeton, NJ, 1965).

[22] C. F. Dong, D. Chernin, Y. Y. Lau, B. W. Hoff, D. H. Simon, P. Wong, G. B. Greening, and R. M. Gilgenbach,
Harmonic content in the beam current in a traveling-wave tube, IEEE Trans. Electron Devices 62, 4285 (2015).

[23] A. L. Eichenbaum, Traveling wave prebunching of electron beams for free electron masers, IEEE Trans. Plasma Sci. 27, 568 (1999).

[24] M. Chodorow, E. L. Ginzton, and E. J. Nalos, Debunching of electron beams constrained by strong magnetic fields, Proc. IRE 41, 999 (1953).

[25] Y. Y. Lau, Theory of Crossed-Field Devices, and a Comparative Study of Other Radiation Sources, High Power Microwave Sources, edited by V. L. Granatstein and I. Alexeff (Artech House, Norwood, MA, 1986). 